\title{
A comparative study of the characteristics of two Coxsackie A virus type 16 strains (genotype $B$ )
}

\author{
YANG ErXia ${ }^{1}$, ZHAO Heng ${ }^{1}$, ZHANG Ying ${ }^{1}$, LIU JianSheng ${ }^{1}$, LIAO Yun ${ }^{1}$, WANG LiChun ${ }^{1}$, \\ CUI PingFang ${ }^{1}$, YANG LiXian ${ }^{1}$, LIU LongDing ${ }^{1}$, DONG ChengHong ${ }^{1,2}$, DONG ShaoZhong ${ }^{2}$, \\ SHAO CongWen ${ }^{2}$, JIANG Li $^{2}$, SUN Le ${ }^{1} \&$ LI QiHan ${ }^{1 *}$ \\ ${ }^{1}$ Institute of Medical Biology, Chinese Academy of Medicine Science, Peking Union Medical College, Yunnan Key Laboratory of \\ Vaccine Research and Development on Severe Infectious Diseases, Kunming 650118, China; \\ ${ }^{2}$ Jiangsu Convax Biotechnology Co., Ltd., Taizhou 225300, China
}

Received February 15, 2012; accepted March 8, 2012

\begin{abstract}
Coxsackie A virus is one of the major pathogens associated with hand, foot and mouth disease (HFMD). The etiological characteristics of Coxsackie A virus type 16 (CA16) are thought to correlate with the pathological process of its infection. Two CA16 strains that were isolated from a severe HFMD patient presented with different plaque forms. This observation, along with biological analysis, indicated that the differences in the strains' biological characteristics, such as proliferation kinetics and immunogenicity, correlate with differences in their pathogenicity toward neonatal mice. Furthermore, these differences are thought to be associated with the sequence of the $5^{\prime}$ non-coding region of the viral genome and the VP1 structural region sequence. The results suggest that the biological and genetic characteristics of the CA16 viral strains are relevant to their pathogenicity.
\end{abstract}

CA16 strains, proliferation kinetics, pathogenicity

Citation: $\quad$ Yang E X, Zhao H, Zhang Y, et al. A comparative study of the characteristics of two Coxsackie A Virus type 16 strains (genotype B). Sci China Life Sci, 2012, 55: 336-342, doi: 10.1007/s11427-012-4313-Z

Hand, foot and mouth disease (HFMD) is responsible for large epidemics in Asia and the Pacific [1-3]. The etiology of this viral disease, which mainly infects children, is unclear. Among the varieties of enteric viruses that could cause HFMD [4-6], EV71 and CA16 are the two major disease-causing pathogens [6,7]. Preliminary pathogen detection in infected cases suggests that EV71 is responsible for $49 \%-52 \%[8,9]$ of reported cases, while CA16 is responsible for approximately $45 \%-48 \%$ [8,9]. Although recent studies indicate that most severe and fatal cases of HFMD are caused by EV71 [10], several in-depth studies have demonstrated that, apart from causing a large number

*Corresponding author (email: imbcams.lq@gmail.com) of ordinary HFMD cases, which behave as typical oral mucosa herpes or hand and foot herpes accompanied by related symptoms, CA16 may also lead to a small number of severe cases. In addition, damage to the central nervous system is a pathological feature of CA16 infection $[11,12]$. Therefore, it is important to further investigate the etiological features of CA16 in the context of the prevention and control of HFMD. Our early work focused on etiology of HFMD epidemics at Fuyang, Anhui, China in 2008. An etiological analysis of CA16 strains from other HFMD epidemiological areas was conducted in the following years [13]. We isolated and collected different CA16 strains from HFMD patients and performed etiological studies. The biological phenotypic characteristics of one CA16 virus sample isolated from a severe 
HFMD patient from Guangxi in 2010 suggested that two phenotypic strains are involved in the tissue-culture process. The two strains present different proliferation kinetics and lesion capacity in sensitive animals. The differences may be related to minor base pair mutations on the $5^{\prime}$ non-coding region, the VP1 region, and the 3 ' non-coding region. Such phenotypic characteristic and genome structural differences may lead to significant differences in the course of CA16 infections and relevant pathological results, e.g., the severity of HFMD pathological changes. Accordingly, these differences require further investigation to determine the CA16-related mechanisms leading to HFMD, the epidemiological characteristics of CA16 infection, and for therapy and vaccine development.

\section{Materials and methods}

\subsection{Cells}

Vero cells (ATCC, qualified for vaccine manufacture) were cultured at $37^{\circ} \mathrm{C}$ in Dulbecco's Modified Eagle's Medium (DMEM) supplemented with 5\% fetal calf serum.

\subsection{Viral isolation and culture}

CA16 virus was isolated from the throat swab of a Guangxi HFMD patient in 2010. The viral samples were diluted in $0.01 \mathrm{~mol} \mathrm{~L}^{-1} \mathrm{PBS}(\mathrm{pH}$ 7.2) and filter-sterilized using a $0.22-\mu \mathrm{m}$ filter (Millipore, Bedford, MA) before being used to infect Vero cells. Samples were collected after the observation of cytopathic effects (CPE), and the viral titer was determined for further studies.

\subsection{Plaque cloning and passaging of viral strains}

Monolayers of Vero cells in a 6-well tissue culture plate were incubated with serially diluted virus preparation for 20 min. The cells were overlaid with nutrient agarose and cultured for 4-5 d. Typical plaques were selected and inoculated into flasks (DMEM Medium containing 5\% FBS). The supernatants were harvested when the CPE reached $95 \%$. After two rounds of plaque cloning, the selected viral clones were inoculated into a monolayer of Vero cells for continuous subcultures.

\subsection{Viral titration}

The viral stocks were serially diluted 10 times using serum-free viral dilution buffer, and $100 \mu \mathrm{L}$ of the diluted stocks were added to each of the 96-well plates, which had previously been coated with $80 \%$-confluent Vero cells. Overall, 8 parallel wells for each dilution stock were used. The 96-well plates were incubated at $37^{\circ} \mathrm{C}$ in a $5 \% \mathrm{CO}_{2}$ incubator for 5-7 d to observe the virus's cytopathicity. The
$50 \%$ cell culture infective dose $\left(\mathrm{CCID}_{50}\right)$ was determined by Reed-Muench assay.

\subsection{Viral cross-neutralization test}

G20-M and G20-D strains obtained from plaque clones were used to immunize mice to prepare specific antiserum. The harvested viral G20-M and G20-D preparations were diluted to $2 \times 10^{3} \mathrm{CCID}_{50} \mathrm{~mL}^{-1}$ with maintaining buffer, and $50 \mu \mathrm{L}$ of the diluted viral stocks was added to each of the 96-well plates. In addition, the anti-G20-M and anti-G20-D serum were serially diluted, i.e., in ratios of $1: 8,1: 16,1: 32$, $1: 64,1: 128,1: 256,1: 512$, and $1: 1024$. Fifty-microliters of the serially diluted anti-G20-M serum was added into each well of the 96-well plates containing the G20-M and G20-D strains. After incubation of the virus and serum together for $2 \mathrm{~h}$, Vero cells were added, before an additional 5-7 d incubation to observe the neutralization effect. The same operation was performed with serially diluted G20-D serum.

\subsection{Neonatal mice test and pathological analysis}

One-day-old specific pathogen free (SPF) level imprinting control region (ICR) neonatal mice (weight 1.8-2.0 g, provided by the Experimental Animal Center, Institute of Medical Biology, Chinese Academy of Medicine Sciences) were randomly divided into blank control, G20-M, and G-20D groups with three litters per group and 10-13 neonatal mice per litter. The neonatal mice in the virus groups were injected intracerebrally with two different viral strains $\left(10^{4} \mathrm{CCID}_{50}\right)$, while the control group mice were injected with PBS. Appetite changes, activities, mental status, limb paralysis, and dying or death symptoms were observed for 7 d post-injection. Dead mice presenting obvious pathological features were sampled. Their brain and lung tissues were taken and fixed in 10\% aldehyde solution for 3-5 d. Neonatal mouse brain tissue lesions were observed using microscopy after dehydration, paraffin embedding, sectioning, hematoxylin/eosin (HE) staining, and cementing.

\subsection{Preparation of immunized animal serum}

Neonatal mice were immunized via abdominal subcutaneous multi-point injection of purified two viral strains $\left(10^{6.5}\right.$ $\mathrm{CCID}_{50}$ ) mixed with Freund's incomplete adjuvant, while the control group was injected with PBS. Mice were immunized through a 0 - and 4-week program. Tail blood collection was followed by two immunizations and serum collection.

\subsection{Viral gene PCR and sequence analysis}

Total RNA was extracted from the harvested viral supernatant and pathological tissues using a TRNzol-A ${ }^{+}$total RNA 
extraction kit (Tiangen, China).

Primers were designed to amplify the $5^{\prime}$ and $3^{\prime}$ ends of the viral genome along with the VP1 region of human Coxsackie virus A16 strain KMM/08 (gi|330375630). PCR amplification was performed using the PremSTAR MAX Premix system (Takara, Japan), following the manufacturer's protocol. PCR sequences of three regions of G20-M and G20-D were obtained and bi-directionally sequenced by TIANGEN. The aligned sequences were analyzed using OMIGA software. The amplification and sequencing were repeated three times.

The PCR primer sequences used were as follows:

G20 5' non-coding region primers: 5' UNRf (1-23) 5'TGAAACAGCCTGTGCCTTGTTC-3', 5' UNRr (835-856) 5'-TATGCATCCTTGTAGTAGTTTA-3';

G20 3' non-coding region primers: 3' UNRf (6981-7004) 5'-AGAACTGGTAAAGAGTATGGATTG-3', 3' UNRr (7397-7416) 5'-GCTATTCTGGTTATAACAAA-3';

VP1 structural protein primers: VP1f (2320-2350) 5'TGTCGTACCCATTGGTGCTCCCACT-3', VP1r (34403470) 5'-AGTGGTGGAAGAGACTAACAAGTCCCTAGA-3'.

For quantitative real-time PCR (qRT-PCR), the One-Step PrimeScript $^{\text {TM }}$ RT-PCR Kit (TaKaRa, Japan) was used according to the manufacturer's protocol. A CA16 nucleic acid standard was serially diluted to $10^{5}, 10^{4}, 10^{3}, 10^{2}$, and 10 copies $\mu \mathrm{L}^{-1}$ and added into the same reaction plate for the PCR reaction. Primers and probes designed using the VP1 conserved region sequences of the G20 strain were as follows:

Vp1f: 5'-CTAGTAGTCACAGATTAGGCACTGGTG-3'; Vp1r: 5'-CATTGTGATGATGCTGACAAGACC-3'; Probe (2411-2438): 5'-FAM-CGTCTAATGCTAGCGACAATAMRA-3'.

\subsection{Analysis of G20-M and G20-D genetic distance}

The viral gene sequences of 9 CA16 strains were obtained from GenBank for analysis of the G20-M and G20-D 5' non-coding region genetic distance. Each selected strain represented a typical epidemical strain in different areas [14-16].

The sequences were analyzed, and an evolutionary tree was drawn using EditSeq and MegAlign in DNAstar.

\subsection{Data analysis}

The G20-D and G20-M proliferation kinetics data were collected three times at $37^{\circ} \mathrm{C}$ and $39.5^{\circ} \mathrm{C}$, before being analyzed statistically. For the statistical analysis, non-parametric tests were used to analyze the data from the $37^{\circ} \mathrm{C}$ experiments. The $P$ value of the infectious titer was 0.00 , and the $P$ value of the qRT-PCR was 0.0058 . Student's $t$-test was used to analyze the $39.5^{\circ} \mathrm{C}$ data. With a $t$ value of -13.2873 , the $P$ value was 0.00 . All $P$ values were $<0.05$ and thus were considered to be statistically significant.

\section{Results}

\subsection{Isolation of two CA16 viral strains and their bio- logical phenotype characteristics}

After a preliminary analysis of more than 40 CA16 viral strains from a variety of areas in mainland China, we chose 31 samples and adopted Vero cells for passaged proliferation. We observed significantly higher titers for G20 proliferation in cells compared with other strains. Phenotypic analysis of harvested viral supernatants of plaques from those Vero cells used to proliferate the strains suggested that there were two different phenotypic G20 strains (Figure 1). One strain presents larger and irregular plaques, and the other presents smaller plaques (Figure 1). Sequence analysis of the $5^{\prime}$ non-coding region, $3^{\prime}$ non-coding region, and VP1 coding region of 10 plaques selected from the two strains confirmed that there were two different strains, which were subsequently named G20-D and G20-M.

\subsection{Proliferation kinetics of two CA16 strains}

Based on the viral isolation and phenotypic analysis results, G20-D and G20-M were proliferated in Vero cells and analyzed for strain proliferation kinetics. The same multiplicity of infection (MOI; 0.05) was used to inoculate Vero cells along with the time sampling for an infectious viral titration test. The results indicated that the G20-D strain has a higher proliferation rate than the G20-M strain in Vero cells. Statistically different values were obtained after $24 \mathrm{~h}$ of infection (Figure 2A). Dynamic infection analysis using qRT-PCR also supported this observation (Figure 2B). To further confirm the proliferation differences of the two strains and their biological characteristics, we took advantage of the fact that different strains of enterovirus proliferate differently under a relatively high incubation temperature [17]. We observed the proliferation kinetics of the two strains at $39.5^{\circ} \mathrm{C}$. The results suggested that G-20 M did not proliferate at $39.5^{\circ} \mathrm{C}$, while significant proliferation

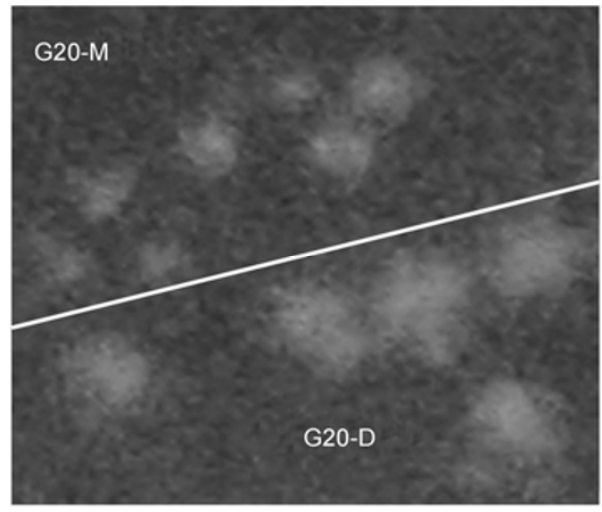

Figure 1 Phenotypic differences between two CA16 strains: G20-D and G20-M. Plaque morphology of strains G20-D (large plaque) and G20-M (small plaque) stained with $0.5 \%$ crystal violet dye. 

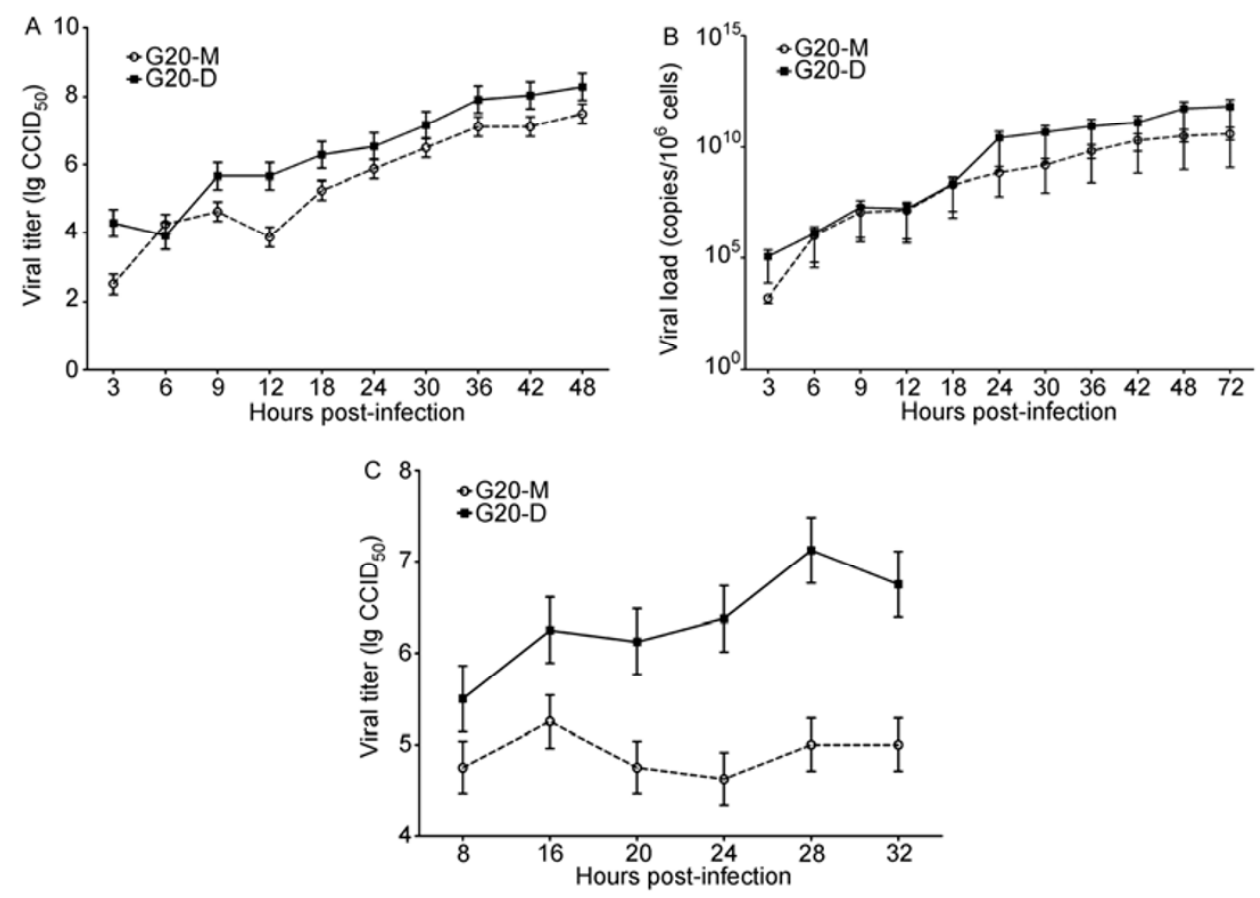

Figure 2 Comparison of infection kinetics of strains G20-D and G20-M on Vero cells. A, Growth kinetics (viral titer) of G20-D and G20-M on Vero cells at $37^{\circ} \mathrm{C}$ (from three biological replicates). B, Growth kinetics (viral load) of G20-D and G20-M on Vero cells at $37^{\circ} \mathrm{C}$ (qRT-PCR repeated three times). C, Growth kinetics (viral titer) of G20-D and G20-M on Vero cells at $39.5^{\circ} \mathrm{C}$ (from three biological replicates). Vero cells were infected by the virus at an MOI of 0.05 . The fluid samples were collected at different time points and viral titer or viral load (genomic copies) were examined by titration or qRT-PCR, respectively.

was observed for the G-20D strain (Figure 2C).

\subsection{Comparison of the pathogenicity of the two strains in neonatal mice}

An earlier study demonstrated that CA16 virus is pathogenic to neonatal mice [18]. This pathogenicity has been used as a phenotype-defining feature of CA16 [18] and as a comparative indicator between different genotypes or strains. Comparing the pathogenicity of the G20-D and G20-M strains would permit observation of fundamental biological features of the two strains. Each of the two strains was intracerebrally injected $\left(10^{4} \mathrm{CCID}_{50}\right)$ into neonatal mice. G20-D was observed to be pathogenic to neonatal mice, with a 7-d post-infection death rate that exceeded $50 \%$ (Figure 3C). The comparative G20-M death rate was lower than $5 \%$ (Figure 3C). The neonatal mice first displayed paralysis of hind limbs, followed by death within one day. Further histological examination of the pathological changes indicated that infection with the G20-D strain might cause dissolution of glial cells, nuclei rupture, intraventricular hemorrhage, and the formation of glial nodules in the brain tissue of neonatal mice (Figure $3 \mathrm{~A}$ and B). Interestingly, G20-D infection in the neonatal mouse brain also causes obvious lung tissue lesions, including the swelling and bleeding of the alveolar cavity, and alveolar tissue destruction (Figure 3D and E). Moreover, viral analysis to ensure such lesions was related to viral infection (viral titra- tion and qRT-PCR), demonstrating that the bleeding brain lesions of G20-D-infected mice were accompanied by viral proliferation $\left(10^{4.5}\right.$ CCID50, Figure $\left.3 \mathrm{~F}\right)$. The tissues that displayed pulmonary congestion and alveolar hemorrhage presented $10^{1.5} \mathrm{CCID}_{50}$ virus (Figure $3 \mathrm{~F}$ ). In contrast, in the G20-M group, only two neonatal mice died among the three infected litters (10-13 mouse per litter). Pathological studies of the brain and lung tissues of the two mice indicated only mild inflammatory cell aggregation in brain tissue, mild hyperemia of the alveolar wall, and infiltration of inflammatory cells (Figure $3 \mathrm{G}$ and $\mathrm{H}$ ). At the same time, $10^{2.5}$ $\mathrm{CCID}_{50}$ of virus was detected in the brain tissue, while no virus was detected in the lung tissue (Figure 3I). These results suggest significant differences between the two strains in terms of animal pathogenicity.

\subsection{Immunogenicity of the two strains and the cross- neutralizing capacity of strain-induced antibodies}

To further understand the biological phenotypes of the G20-D and G20-M strains, we used identical amounts of virus $\left(10^{6.5} \mathrm{CCID}_{50}\right)$ mixed with Freund's incomplete adjuvant to immunize mice by intramuscular injection. Serum was sampled after two immunizations with a 0 and 4 week program. Serum neutralization titer was measured using 100 $\mathrm{CCID}_{50}$ viral doses. The results indicated that the two strains induced neutralized antibodies with different titers against the inducing viral strain. When neutralizing the other 

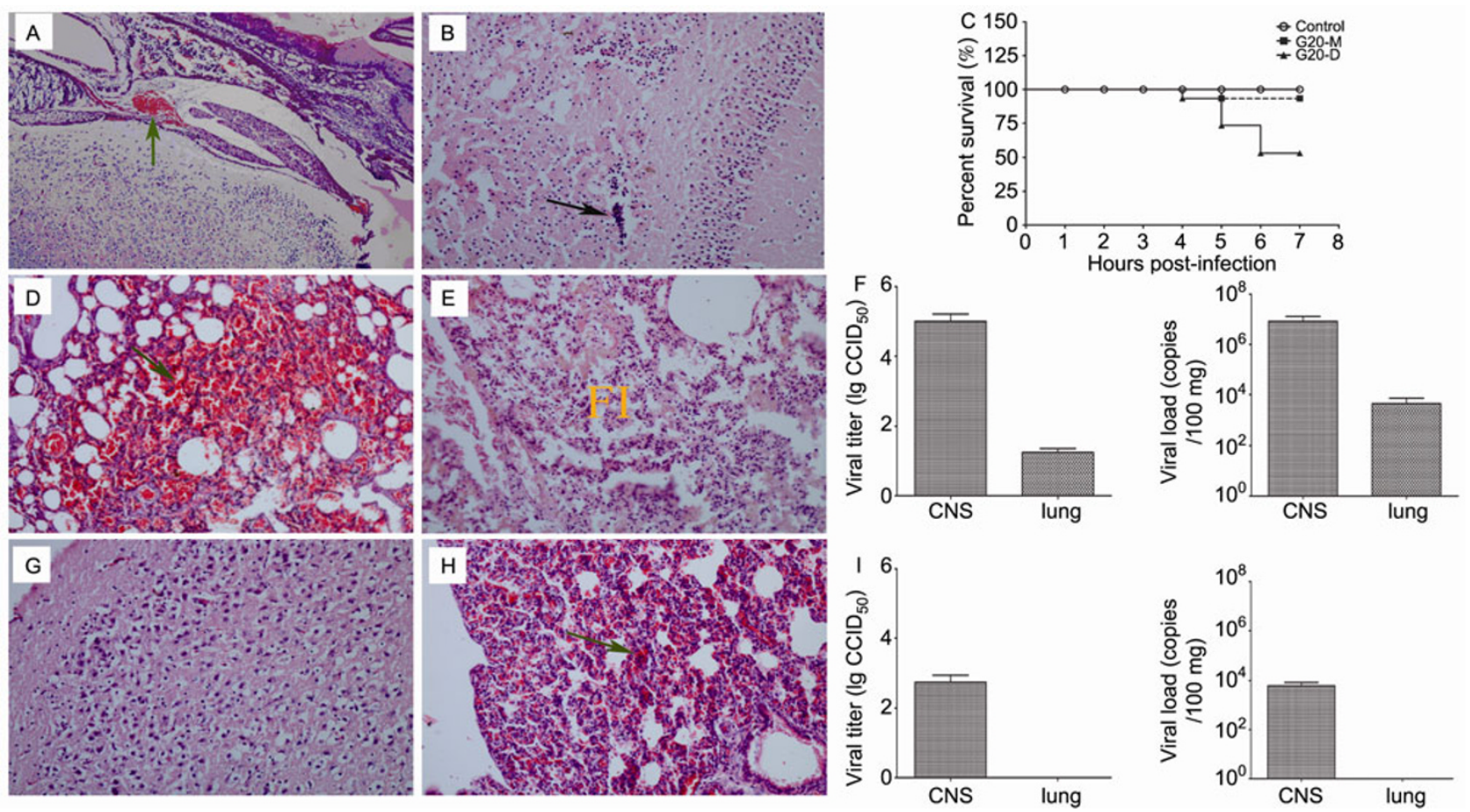

Figure 3 Analysis of pathogenicity of G20-D and G20-M in neonatal mice. A and B, Pathological changes induced by G20-D in neonatal brain tissue ( $\times 200)$. Intraventricular hemorrhage and the formation of glial nodules are indicated by a green arrow and a black arrow, respectively. C, Comparison of induced death rate in neonatal mice infected by G20-D and G20-M. D and E, Pathological changes induced by G20-D in neonatal lung tissue $(\times 200)$. Hyperemia and hemorrhage of alveolar wall and cavity and mild autolysis of the alveolar tissue (FI) are indicated by a green arrow and a black arrow, respectively. F, Virus titration of brain and lung tissues of G20-D lethal neonatal mice. Titration by tissue culture and qRT-PCR were performed to examine the brain and lung tissues of 30 lethal neonatal mice. G, Pathological changes of the brain tissue in G20-M lethal neonatal mice $(\times 200)$, showing mild inflammation cell aggregation. H, Pathological changes of lung tissue in G20-M lethal neonatal mice $(\times 200)$, showing mild hyperemia of the alveolar wall and some inflammatory cell filtration (green arrow). I, Virus titration of brain and lung tissue in G20-M lethal neonatal mice. Brain and lung tissues from two mice were sampled and examined by viral titer. B-E, G, and $\mathrm{H}$ are $\mathrm{HE}$ staining results.

strain using the same titer, the strains displayed significant differences. Among these differences, the G20-D-induced neutralization antibody showed a $1: 16$ neutralization titer to G20-D, but 1:512 to G20-M for the same dosage of 100 $\mathrm{CCID}_{50}$. Conversely, the G20-M-induced neutralization antibody presented a $1: 16$ neutralization titer to the G20-M strain and a 1:8 to the G20-D strain. The results indicate the two strains have significant differences in their immunogenicity.

\subsection{Comparison of gene sequences of the two pheno- typically different strains}

Based on the analysis of different biological features of G20-D and G20-M and according to the basic principles of biological characteristics and related gene sequences of enteroviruses [19-21], we decided to sequence the $5^{\prime}$-noncoding region, the $3^{\prime}$ non-coding region, and the VP1 coding region. We repeated the sequencing three times and aligned the sequence (Figure 4A). Comparison of the three gene regions from the two strains revealed individual nucleotide difference in all three regions. Seven different nucleotides were observed within the $5^{\prime}$ non-coding region (Figure 4A). The G20-D strain has an A at position 33, but the G20-M has a G, while the same difference is observed at site 35. G20-D has a $\mathrm{T}$ at position 36, while G20-M has a G. There is a $\mathrm{T}$ at position 37 in G20-D but an A in G20-M. There is a nucleotide missing at position 44 of G20-D. In addition, G20-D has a T, while G20-M has a $\mathrm{C}$ at position 110, and at position 212, G20-D has a C and G20-M has a T. Three-nucleotide differences were observed in the $3^{\prime}$ non-coding regions (Figure 4A). At position 52, G20-D has a C, but G20-M has a G. There is one nucleotide missing at position 90 for G20-D, and at position 312, G20-D has a T, but G20-M has a $\mathrm{C}$. The sequence analysis of the VP1-coding region indicated that nucleotide differences led to three amino acid differences between the two strains (Figure 4A). G20-D has an M, but G20-M has a T at amino acid 98. At amino acid 240, G20-D has a T, while G20-M has an I. At amino aid 248, G20-D has a T, but G20-M has an A. The nucleotide differences in both the coding and non-coding regions (Figure 4A) suggest that the biological differences between the two strains are based on their genetic structures. In addition, these strains' sequences provide clues for further studies of loci related to proliferation efficiency, pathogenicity to animals, and immunogenicity between different strains. The evolutionary tree drawn using VP1 and the $5^{\prime}$ non-coding region indicates that, although 
$\underline{A}$

\begin{tabular}{cccc}
\hline & $\begin{array}{c}\text { Site of different base } \\
\text { or amino acid }\end{array}$ & G20-M & G20-D \\
\hline 5' UTR & 33 & G & A \\
35 & G & A \\
36 & G & T \\
37 & A & T \\
44 & G & - \\
110 & C & T \\
212 & T & C
\end{tabular}

B

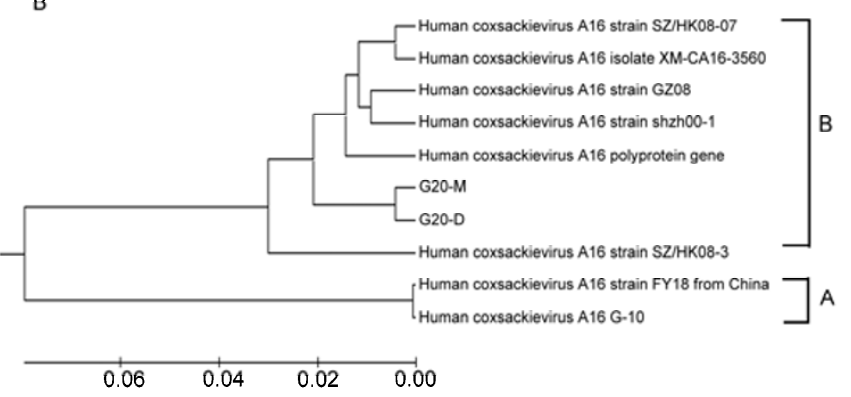

\begin{tabular}{lccc} 
3' UTR & 52 & G & C \\
& 90 & A & - \\
& 312 & C & T \\
\hline VPI & 98 & & \\
& 240 & T & M \\
& 248 & I & T \\
& & A & T \\
\hline
\end{tabular}

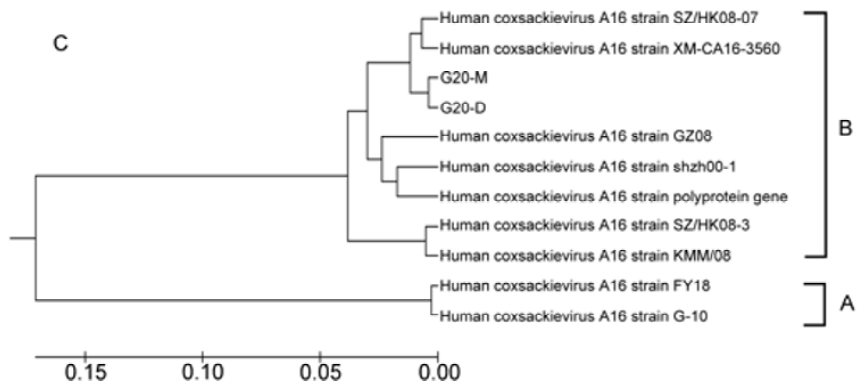

Figure 4 Sequence comparison of 5'-end, 3'-end, and VP1 coding region in strains G20-D and G20-M. A, Comparison of the 5'- non-coding region, 3'non-coding region, and amino acid sequences of VP1 structural protein between G20-M and G20-D (each of the three regions within the two strains was sequenced three times independently and aligned with the same result). B, Phylogenetic analysis of G20-M and G20-D, based on the sequence of CA16 5'non-coding region. C, Phylogenetic analysis of G20-M and G20-D, based on the sequence of CA16 VP1.

the two strains have only a few differences, they both belong to different sub-genotypes of the B genotype (Figure $4 \mathrm{~B}$ and $\mathrm{C})$.

\section{Discussion}

Early studies of enteroviruses showed that as an enterovirus, CA16 is pathogenic to humans [22,23]. CA16 can cause herpes pharynx inflammation, HFMD, and viral encephalitis under certain conditions [12,24]. The virus has three genotypes: A, B and C [9]. Relevant epidemiological analyses suggest that the main phenotype in China is B [16]. Increasing attention is being paid to CA16 as a pathogenic cause of HFMD pandemics, in addition to EV71, in recent studies $[25,26]$. Several reports indicate that CA16 causes mild HFMD and is responsible for general hand, foot and mouth herpes and flu-type symptoms [27]; however, other reports suggest that CA16 can cause severe cases and can affect the nervous system. However, the mechanism of infection and pathological consequences remain unclear [28]. Therefore, investigating the biological characteristics of different CA16 strains from an etiological point of view could aid in understanding the differences between diseases caused by the virus and provide information concerning its pathological mechanisms.

The two different strains described in this paper are from the throat swab secretions from the same patient. Although our observation of the viral biological features is based on second-generation virus proliferated in Vero cells, the source of the two strains might be genetic mutations, either from the same infected individual or from in vitro proliferation. Although we have not proven either of the two possibilities or their related infectious biological mechanisms to date, the comprehensive comparison of the two strains still clearly showed that even within the same genotype, there are strains that present different biological characteristics. The differences are based on genetic structures within the 5' and $3^{\prime}$ non-coding regions and VP1 coding regions and are responsible for the observed proliferation kinetics differences. Previous data suggested that specific sequence elements on enterovirus $5^{\prime}$ and $3^{\prime}$ non-coding regions are essential for regulation of viral proliferation. Studies on poliovirus have shown that a change in the 480-nucleotide region can directly affect the pathogenicity of the virus [29]. In our work, seven nucleotide changes in the $5^{\prime}$ non-coding region and the three in the $3^{\prime}$ non-coding region between G20-D and G20-M were not same as those detailed in previous studies. It is unclear if these sequences are essential regulatory elements for viral proliferation in the CA16 viral genome; however, our study provided a sound basis for further studies. In addition, the differences in immunogenicity of the two strains and changes at specific sites in the VP1 coding regions also provide a foundation for further study of the immunogenicity and gene regulatory sites of CA16. Interestingly, our observations of the changes in gene sequences could lead to obvious differences in the formation of animal lesions by specific virus strains. Intracerebral injection of the G20-D strain in neonatal mice not only caused obvious damage to neuronal cells in the brain, but also caused the formation of glial nodules that lead to paralysis of the hind limbs. In the infection process, conges- 
tion and edema of lung tissues filled the alveolar space with secretions, resulting in loss of function. Although we do not know the direct cause of death of the neonatal mice from pathological examinations, the severe congestion and edema in the alveolar space obviously cannot be ignored. Viral tests of brain and lung tissue suggested that the latter had only a very low viral load. These results suggest the direct cause of death in severe HFMD patients infected by CA16 and EV71 is neurogenic pulmonary edema and loss of cardiopulmonary functions [30]. If the connection is confirmed, it will clarify the pathological manifestations of mild and severe HFMD from an etiological point of view. These conclusions are based on the possibility, as observed in CA16 infectious epidemiological studies that elicited no obvious serum antibody response [31], that etiological evidence was still presented. In addition, these results will aid CA16 vaccine research and development. The biological characteristics of the two strains observed in this study might relate to the structural differences caused by gene sequence differences. However, the direct relationship between the specific gene sequences and the related symptom changes is uncertain, although the data does provide clues for further research. Indeed, additional studies are underway, using mutant virus sequences to locate CA16 biological features, especially those that relate to changes in animal pathogenicity.

This work was supported by the National Basic Research Program of China (Grant No. 2011CB504903), the National Natural Science Foundation of China (Grant No. 81171573), the Important National Science \& Technology Specific Projects (Grant No. 2009ZX10004-308) and the General Program of Applied Basic Research Programs Commission, Foundation of Yunnan Province (Grant No. 2011FB116).

1 Chan L G, Parashar U D, Lye M S, et al. Deaths of children during an outbreak of hand, foot, and mouth disease in Sarawak, Malaysia: clinical and pathological characteristics of the disease. Clin Infect Dis, 2000, 31: 678

2 Shimizu H, Utama A, Yoshii K, et al. Enterovirus 71 from fatal and nonfatal cases of hand, foot and mouth disease epidemics in Malaysia, Japan and Taiwan in 1997-1998. Jpn J Infect Dis, 1999, 52: 12

3 July A M J. Deaths among children during an outbreak of hand, foot, and mouth disease-Taiwan, Republic of China, April-July 1998. MMWR Morb Mortal Wkly Rep, 1998, 47: 629-632

4 Wang L H, Li J. Research progress of epidemiology and etiology of hand, foot and mouth disease. Chin Health Serv Manage, 2010, 6: $419-521$

5 Sun J L, Zhang J. Research progress of epidemiology of hand, foot and mouth disease. Chin J Epidemiol, 2009, 9: 973-976

6 Chan K P, Goh K T, Chong C Y, et al. Epidemic hand, foot and mouth disease caused by human enterovirus 71, Singapore. Emerg Infect Dis, 2003, 9: 78

7 Wang Z G, Liu X L, Yang T T, et al. Etiology of hand, foot and mouth disease in Qingdao during 2008-2009. Chin J Virol, 2011, 27: 438

8 Jia L, Zhao C, Zhang L. Comparisons of epidemiological and clinical characteristics in children with hand foot mouth disease caused by Enterovirus 71 and Coxackievirus A16. CJCP, 2011, 08: 635-637

$9 \mathrm{Li} \mathrm{L}, \mathrm{He} \mathrm{Y}$, Yang H, et al. Genetic characteristics of human enterovirus 71 and coxsackievirus A16 circulating from 1999 to 2004 in
Shenzhen, People's Republic of China. J Clin Microbiol, 2005, 43: 3835

10 Zhang Y, Cui W, Liu L, et al. Pathogenesis study of enterovirus 71 infection in rhesus monkeys. Lab Invest, 2011, 91: 1337-1350

11 Yang Z H, Zhu Q R, Li X Z, et al. Detection of enterovirus 71 and coxsackievirus A16 from children with hand, foot and mouth disease in Shanghai, 2002. Chin J Pediatrics, 2005, 43: 648-652

12 Chang L Y, Lin T Y, Huang Y H, et al. Comparison of enterovirus 71 and coxsackievirus A16 clinical illnesses during the Taiwan enterovirus epidemic, 1998. Pediatr Infect Dis J, 1999, 18: 1092

13 Li R, He H, Zhao X. Etiology analysis and the clinical characteristics of 1073 children with hand-food-mouth disease. Beijing Med J, 2011, 09

14 Ma S H, Chen J Y, Li H, et al. Genetic characters of Coxsackievirus A16 strain in 2009 in Kunming Region, Yunnan province China. Chin J Biol, 2012, 2: 9-12

15 Yip C C Y, Lau S K P, Zhou B, et al. Emergence of enterovirus 71 "double-recombinant" strains belonging to a novel genotype D originating from southern China: first evidence for combination of intratypic and intertypic recombination events in EV71. Arch Virol, 2010, 155: 1413-1424

16 Zong W, He Y, Yu S, et al. Molecular Phylogeny of Coxsackievirus A16 in Shenzhen during 2005-2009. J Clin Microbiol, 2011, 4: 1659

17 Liu Y X. Proliferation dynamics research of EV71 different strains in different temperature condition. Dissertation for Doctoral Degree. Kunming: Kunming Medical University, 2010

18 Dai G Z. Coxackievirus. Microbiology, 1977, 4: 49-51

19 Puenpa J, Theamboonlers A, Korkong S, et al. Molecular characterization and complete genome analysis of human enterovirus 71 and coxsackievirus A16 from children with hand, foot and mouth disease in Thailand during 2008-2011. Arch Virol, 2011: 1-7

20 Zhou F, Kong F, Wang B, et al. Molecular characterization of enterovirus 71 and coxsackievirus A16 using the 5' untranslated region and VP1 region. J Med Microbiol, 2011, 60: 349-358

21 Brown B, Oberste M S, Maher K, et al. Complete genomic sequencing shows that polioviruses and members of human enterovirus species $\mathrm{C}$ are closely related in the noncapsid coding region. J Virol, 2003, 77: 8973-8984

22 Kadurugamuwa J L, Shaheen E. Inactivation of human enterovirus 71 and coxsackie virus A16 and hand, foot, and mouth disease. American J Infect Control, 2011, 39: 788-789

23 Yang F, Zhang T, Hu Y, et al. Survey of enterovirus infections from hand, foot and mouth disease outbreak in China, 2009. Virol J, 2011, 8: 508

24 Goto K, Sanefuji M, Kusuhara K, et al. Rhombencephalitis and coxsackievirus A16. Emerg Infect Dis, 2009, 15: 1689

25 Tan E L, Tak Kwong Chow V, Quak S H, et al. Development of multiplex real-time hybridization probe reverse transcriptase polymerase chain reaction for specific detection and differentiation of Enterovirus 71 and Coxsackievirus A16. Diagn Microbiol Infect Dis, 2008, 61: 294-301

26 Zhao K, Han X, Wang G, et al. Circulating coxsackievirus A16 identified as recombinant type A human enterovirus, China. Emerg Infect Dis, 2011, 17: 1537-1540

27 Ang L W, Koh B K W, Chan K P, et al. Epidemiology and control of hand, foot and mouth disease in Singapore. Ann Acad Med Singapore, 2009, 38: 106-112

28 Wang C Y, Li Lu F, Wu M H, et al. Fatal coxsackievirus A16 infection. Pediatr Infect Dis J, 2004, 23: 275

29 Kawamura N, Kohara M, Abe S, et al. Determinants in the 5'noncoding region of poliovirus Sabin 1 RNA that influence the attenuation phenotype. J Virol, 1989, 63: 1302

30 Yi R S. Clinical analysis of 9 cases of severe hand, foot and mouth disease combined encephalitis . J YouJiang Med College Nationalities, 2009, 31: 66-67

31 Yang Z H, Zhu Q R, Li X Z. Detection of enterovirus 71 and coxsackiev irus A16 from children with hand, foot and mouth disease in Shanghai, 2002. Chin J Pediatr, 2005, 43: 648-652

Open Access This article is distributed under the terms of the Creative Commons Attribution License which permits any use, distribution, and reproduction in any medium, provided the original author(s) and source are credited. 